\title{
Evaluation of sugar yeast consumption by measuring electrical medium resistance
}

\author{
Martín L. Zamora ${ }^{1,2,3}$, Gabriel A. Ruiz ${ }^{1}$ and Carmelo J. Felice ${ }^{1,2}$ \\ 1. Laboratorio de Medios e Interfases, Departamento de Bioingeniería, Facultad de Ciencias Exactas e Ingeniería, Universidad Nacional \\ de Tucumán, Tucumán, Argentina. \\ 2. Instituto Superior de Investigaciones Biológicas (INSIBIO), Consejo Nacional de Investigaciones Cientificas y Técnicas (CONICET), \\ Argentina. \\ 3. E-mail any correspondence to: mzamora@herrera.unt.edu.ar
}

\begin{abstract}
The real-time monitoring of alcoholic fermentation (sugar consumption) is very important in industrial processes. Several techniques (i.e., using a biosensor) have been proposed to realize this goal. In this work, we propose a new method to follow sugar yeast consumption. This novel method is based on the changes in the medium resistance $(\mathrm{Rm})$ that are induced by the $\mathrm{CO}_{2}$ bubbles produced during a fermentative process. We applied a $50-\mathrm{mV}$ and $700-\mathrm{Hz}$ signal to $75 \mathrm{ml}$ of a yeast suspension in a tripolar cell. A gold electrode was used as the working electrode, whereas an $\mathrm{Ag} / \mathrm{AgCl}$ electrode and a stainless-steel electrode served as the reference and counter electrodes, respectively. We then added glucose to the yeast suspension and obtained a $700 \%$ increase in the Rm after 8 minutes. The addition of sucrose instead of glucose as the carbon source resulted in a $1200 \%$ increase in the $\mathrm{Rm}$. To confirm that these changes are the result of $\mathrm{CO}_{2}$ bubbles in the fermentation medium, we designed a tetrapolar cell in which $\mathrm{CO}_{2}$ gas was insufflated at the bottom of the cell and concluded that the changes were due to $\mathrm{CO}_{2}$ bubbles produced during the fermentation. Consequently, this new method is a low-cost and rapid technology to follow the sugar consumption in yeast.
\end{abstract}

Keywords: Yeast, sugar consumption, monitoring, $\mathrm{CO}_{2}$, medium resistance

\section{Introduction}

Yeast-catalyzed alcoholic fermentation (sugar consumption) is a crucial step in industrial processes (food and biofuel) that results in the transformation of sugars (glucose and fructose) into alcohol and carbon dioxide [1]. The yeast Saccharomyces cerevisiae has been known for thousands of years and is routinely used in many traditional biotechnological processes, including bread making and the production of several alcoholic beverages [2].

A variety of techniques have been proposed for monitoring the sugar consumption by yeast, such as the measurement of the amount of $\mathrm{CO}_{2}$ released during the process using electrochemical $\mathrm{CO}_{2}$ probes [3] , optical fiber refractometry, density determination using flexural oscillators, and ultrasound techniques [4].

In addition, Piermarini et al. described a method for monitoring the consumption of glucose and fructose in real time [1]. However, the disadvantage of this method is that it requires the construction of a biosensor that uses glucose oxidase as a bio-receptor. The production of both carbon dioxide and alcohol is a consequence of the biological activity of cells during the fermentation step. The online measurement of carbon dioxide in liquid (grape juice to wine) can be conducted by measuring changes in the medium resistance as a result of $\mathrm{CO}_{2}$ bubbles. Thus, the fermentation kinetics could be controlled (or monitored) by the measurement of the total carbon dioxide [5]. It is known that carbon dioxide bubbles increase the value of the medium resistance [6].

In 2009, Perez et al. described a method for the realtime monitoring of the fermentation of sugar by yeast cells. These researchers measured changes in the medium resistance $(\mathrm{Rm})$, which is defined as the resistance of a certain amount of electrolyte solution some distance from the working electrode and the reference electrode (Ruiz, G., Ph.D thesis, Universidad Nacional de Tucumán, Tucumán, Argentina, 2010); the changes in the resistance would be the result of the $\mathrm{CO}_{2}$ produced during the fermentation.

The main disadvantages of the technique developed by Perez and colleagues are the following. First, it is an offline method, which implies that it is necessary to extract medium from the fermentation tank using a peristaltic pump. In addition, their method requires the use of temperature compensation because the temperature is not controlled during the assay. The measurement time using our technique is 80 minutes, whereas the duration of the experiments conducted by Perez and co-workers was $200 \mathrm{~h}$, which allowed the growth and fermentation of the yeast. The duplication time of Saccharomyces cerevisiae is approximately $90 \mathrm{~min}$ in rich medium [7]. In the experiments presented in this paper, we use a salt medium; thus, the duplication time is closer to 90 minutes, which means that the yeast cells do not grow significantly in our assays. Moreover, the medium used in our assays contains only salts; therefore, yeast cells will not grow because the medium does not contains nitrogen sources and other necessary compounds.

Also, Perez and co-workers used a relatively high frequency to measure $\mathrm{Rm}$, which increases the signal-tonoise ratio. 
In this work, we propose a method to follow the sugars consumption by yeast.

As detailed above, the yeast does not exhibit significant growth during our assay time, which implies that our assay monitors the speed at which the yeast cells consume sugars. The fermentation is followed by the measurement of the changes in the medium resistance (Rm). We used Baker's yeast (Levex) resuspended in a salt medium as the biological material in our assays.

The Rm was measured at $700 \mathrm{~Hz}$ and $50 \mathrm{mV}$ with a cell in a tripolar configuration using a gold electrode as the working electrode. This novel method improves the existent technologies to monitor the fermentation progress using the amount of $\mathrm{CO}_{2}$ produced because it only uses a gold electrode, which economizes the process [5]. In addition, the assay is performed at a low frequency, which decreases the measurement noise. Thus, the objective of this work is to follow the sugar consumption in yeast measuring the effect of the bubbles produced by yeast in the electrical medium resistance.

\section{Materials and methods}

\subsection{Microbiological preparation}

There were two different mediums used in the assay:

1. Saline medium (SM), which consists of $20 \mathrm{mM}$ $\mathrm{KH}_{2} \mathrm{PO}_{4}, 30 \mathrm{mM} \mathrm{KCl}$, and $1 \mathrm{mM} \mathrm{MgCl}$ (Cicarelli, Reagents S.A. San Lorenzo, Santa Fe, Argentina) and has a $\mathrm{pH}$ of 6.5 .The saline medium is used to keep the yeast on a basal metabolic state, so that by adding glucose or sucrose, the only activity will correspond to the metabolism of these sugars.

2. Yeast cell suspension (CS). The CS was obtained by first dissolving $3.5 \mathrm{~g}$ of dry yeast Levex ${ }^{\circledR}$ (Levex, Buenos Aires, Argentina) in $15 \mathrm{ml}$ of SM and stirring the mixture for $5 \mathrm{~min}$ with a magnetic stirrer. The solution was then centrifuged for $10 \mathrm{~min}$ at $5000 \mathrm{rpm}$, and the supernatant was discarded. The cells were then resuspended in $15 \mathrm{ml}$ of $\mathrm{SM}$, and the solution was stirred magnetically. This process was repeated 4 times to ensure the complete removal of biological material ions.

\subsection{Measurement System}

The electrochemical measurements were performed using a Solartron 12508W impedance analyzer (Solartron Group Ltd. Victoria Road, Farnborough Hampshire England) composed of a Solartron 1287 Electrochemical Analyzer and a Solartron 1250 Frequency Response Analyzer with the software provided by the manufacturer (ZPlot ${ }^{\circledR}$, Scribner Associates Inc.).

\subsection{Polishing of the electrodes}

The electrodes were gradually polished in a series of steps and with different roughness degrees: First the electrode was polished with Diamond pasta Praxis ${ }^{\circledR}$ with a roughness of 6 um, then with Diamond pasta Praxis ${ }^{\circledR}$ with a roughness of 3 um and last with alumina powder with a roughness of $1 \mathrm{um}$. The size of the grain matches the European standards (FEPA standard 43-GB-1984; R1993) $[8]$.

\subsection{Electrochemical Cells}

Two different cells were used in this work. A tripolar cell, which is composed of a working electrode (WE), an $\mathrm{Ag} / \mathrm{AgCl}$ reference electrode (Re1) and an AISI 304 stainless-steel concave counter-electrode (CE) with a diameter of $85 \mathrm{~mm}$, was used (Fig. 1). The WE is a solid cylinder (1.5 $\mathrm{cm}$ long), and only $1 \mathrm{~cm}^{2}$ of its transversal section is exposed and coated with gold (the rest was insulated with Grilon). The area of the CE was larger than that of the working electrode to minimize its impedance.

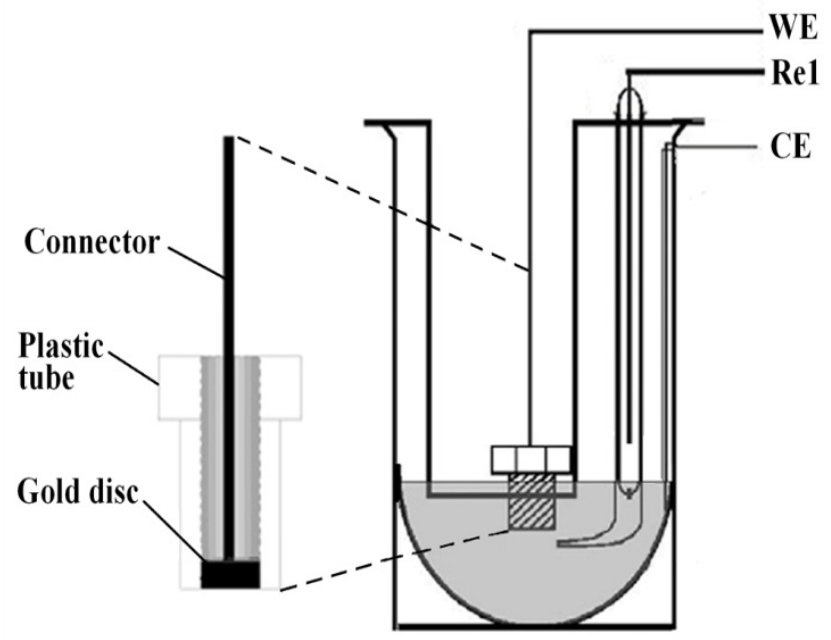

Fig. 1: Tripolar cell (WE: working electrode, Re1: reference electrode and CE: counter electrode).

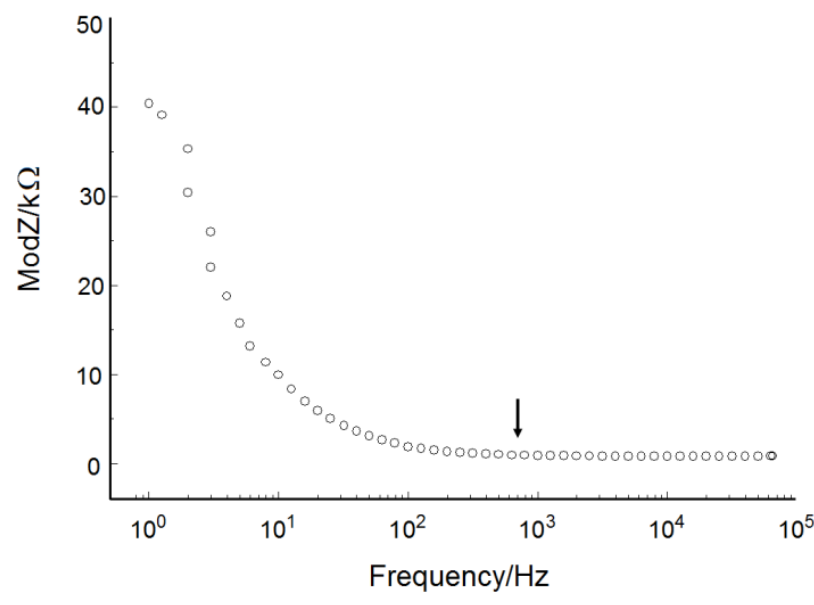

Fig. 2: Magnitude of $\mathrm{Z}$ vs. frequency. The black arrow indicate the working frequency 
An AC signal of $50 \mathrm{mV}$ with a frequency of $700 \mathrm{~Hz}$ was applied to the electrochemical cell. We used $700 \mathrm{~Hz}$, because at this frequency the medium total resistance is equal to $\mathrm{Rm}$ ( $\mathrm{Rm}$ is the medium resistance between $\mathrm{Re} 1$ and $\mathrm{CE}$ ) and the interface is negligible (Fig. 2).

To determinate the effect of $\mathrm{CO}_{2}$ bubbles on the medium resistance, we designed a tetrapolar cell with a length of $28 \mathrm{~cm}$, a width of $2 \mathrm{~cm}$ and four stainless steel electrodes (Fig. 3).

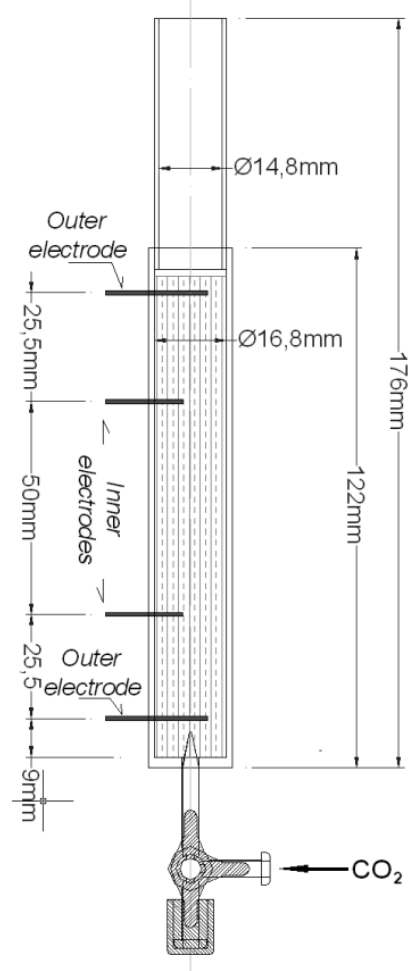

Fig. 3: Tetrapolar cell used in the carbon dioxide bubbling experiments. The $\mathrm{CO} 2$ was insufflated at the bottom of the cell.

\subsection{Monitoring of the sugar consumption}

First, $95 \mathrm{ml}$ of the biological suspension was placed in the previously described tripolar cell. An AC signal of $50 \mathrm{mV}$ with a frequency of $700 \mathrm{~Hz}$ was then applied to the cell. Fifteen minutes after the start of the experiment, $85 \mathrm{mM}$ glucose was added. The same experiment was repeated using sucrose as the carbon source. In the control experiment, we added glucose to the BS medium in the absence of yeast. All experiments were performed at room temperature $\left(25 \pm 0.1^{\circ} \mathrm{C}\right.$ throughout the assay time).

\subsection{Monitoring of the sugar consumption using a blood glucose meter}

This experiment was conducted to calibrate the developed method. The glucose concentration was determined using a Prodigy Autocode blood glucose meter (Diagnostic Device, Inc. Miami, Florida, USA). This measurement of the glucose concentration and the bioimpedance monitoring of the sugar metabolism were performed simultaneously.

\section{$2.7 \mathrm{CO}_{2}$ bubbling}

We performed this experiment to demonstrate that the changes in the $\mathrm{Rm}$ were due to the presence of $\mathrm{CO}_{2}$ bubbles. First, $25 \mathrm{ml}$ of the biological sample was placed in the previously described tetrapolar cell; no carbon source was used in this experiment. The $\mathrm{CO}_{2}$ gas was injected at the bottom of the cell from a high-pressure cylindrical vessel. The experiment was conducted at $20 \mathrm{kHz}$ in a bipolar configuration. At this frequency, all of the variations in the cell are reflected in the medium resistance (Rm); thus, we expected changes in the $\mathrm{Rm} \%$ as a result of the bubbling.

All of the experiments were conducted at $25^{\circ} \mathrm{C}$.

\section{8 Addition of glucose to the base salt medium}

Glucose $(85 \mathrm{mM})$ was added to the base solution in the absence of yeast to demonstrate that the yeast fermentation is responsible for the changes in the $\mathrm{Rm} \%$. The composition of the base solution and the concentrations of yeast and glucose were the same as those used in the previous experiments.

\subsection{Plate Count}

The plate count technique was performed in YPS agar (Yeast extract, Peptone, Sucrose) medium with the following concentration: Yeast extract $10 \mathrm{mg} / \mathrm{L}$; Peptone 20 $\mathrm{mg} / \mathrm{L}$, Sucrose $20 \mathrm{mg} / \mathrm{L}$ and Agar 1,5\%.

\section{Results}

\subsection{Monitoring of the sugar consumption}

Fig. 4 shows a plot of the $\mathrm{Rm} \%$ as a function of time.

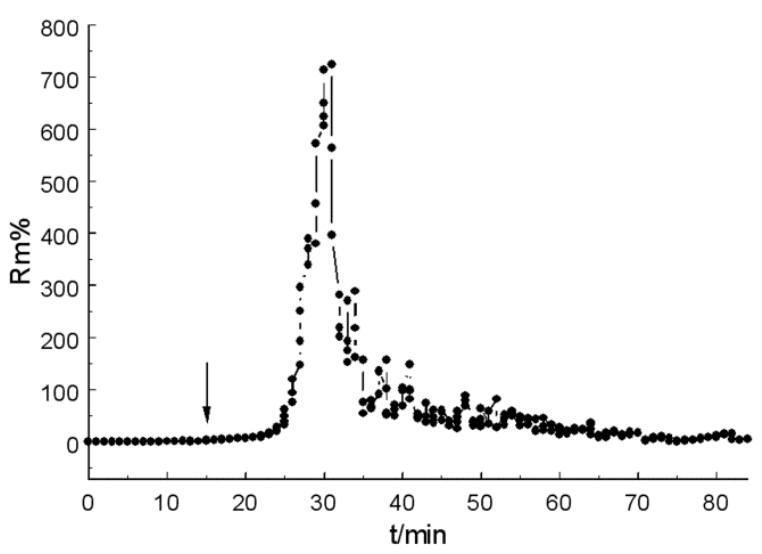

Fig. 4: Plot of the Rm\% as a function of time using glucose as the carbon source. The black arrow indicates the addition of glucose.

The glucose was added $15 \mathrm{~min}$ after the beginning of the experiment. Approximately $7 \mathrm{~min}$ after the addition of 
glucose, the $\mathrm{Rm} \%$ began to gradually increase to $700 \%$. The $\mathrm{Rm} \%$ then started to decrease until it reached approximately the value observed prior to the glucose addition. The time interval during which this increase and decrease occurred is approximately $14 \mathrm{~min}$. We then performed the same experiment using sucrose as the carbon source. Fig. 5 shows the plot of the resulting $\mathrm{Rm} \%$ as a function of time.

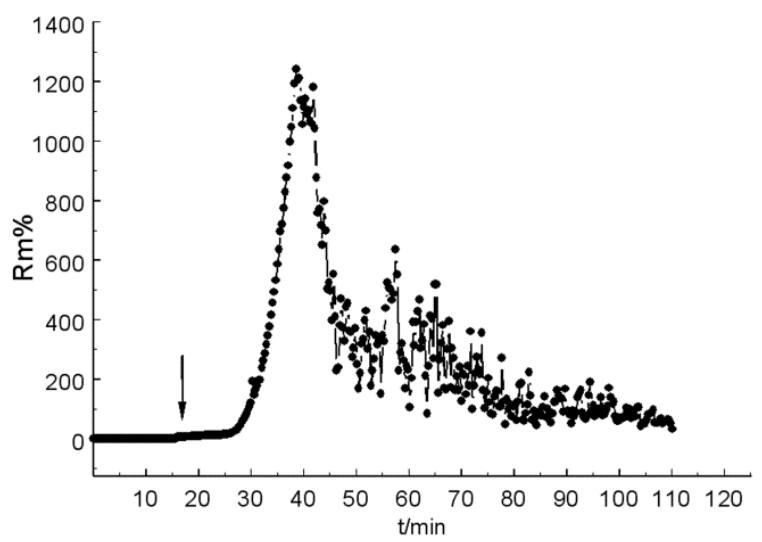

Fig. 5: Plot of the Rm\% as a function of time using sucrose as the carbon source. The black arrow indicates the addition of sucrose.

The sucrose was added 16 min after the beginning of the experiment. Approximately $10 \mathrm{~min}$ after the addition of sucrose, the $\mathrm{Rm} \%$ increased to approximately $1200 \%$. As shown, the maximum $\mathrm{Rm} \%$ is reached after 15 minutes, whereas the maximum $\mathrm{Rm} \%$ is attained within 8 minutes when glucose was used as the carbon source (Fig. 3). In addition, the time interval from the time at which the $\mathrm{Rm} \%$ begins to increase to the end of its decrease is approximately 24 minutes when sucrose is used as the carbon source, whereas the corresponding time interval using glucose as the carbon source is 17 minutes.

Fig. 6 shows the derivative of $\mathrm{Rm} \%$ for glucose and sucrose.

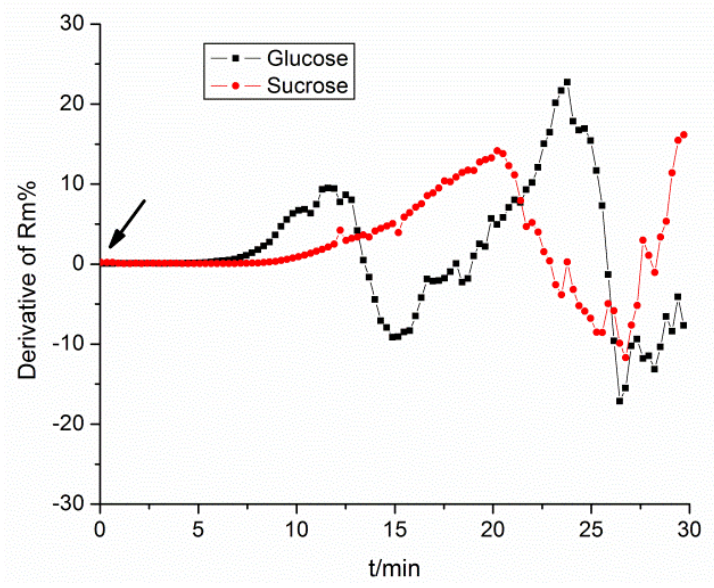

Fig. 6: Comparison of the derivative of $\mathrm{Rm} \%$ for glucose and sucrose.

The sugar was added at $\mathrm{t}=0$. We only consider the first 10 min, since the addition of sugar, in the comparison of the derivatives because there is a large amount of noise in the measurements obtained after this time point as a result of the bubbles. The derivative plots indicate the velocity at which $\mathrm{Rm} \%$ changes as a result of the effects of $\mathrm{CO}_{2}$ bubbles over the electrode [7]. An 11-point derivate algorithm was applied.

Fig. 6 shows that the glucose began to be consumed within 6 min after the start of the experiment, whereas the sucrose consumption started after $8 \mathrm{~min}$. However, the sucrose assay exhibited a higher consumption rate than the glucose experiment. The addition of glucose to the SM demonstrated that the observed changes in $\mathrm{Rm} \%$ were due to the yeast-catalyzed fermentation of the sugar. Fig. 7 shows the corresponding plot of $\mathrm{Rm} \%$ as a function of time.

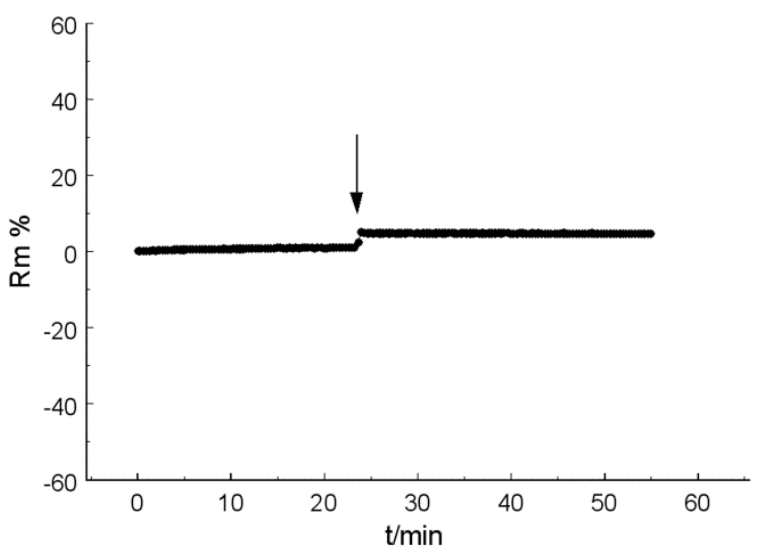

Fig. 7: Plots of the $\mathrm{Rm} \%$ as a function of time in the absence of yeast. The black arrow indicates the addition of glucose.

As shown, the changes in $\mathrm{Rm} \%$ after glucose was added were approximately $1 \%$. Thus, these changes are negligible compared with the changes in the Rm\% observed in the CS after the addition of glucose $(700 \%)$ or sucrose $(1200 \%)$.

\section{$3.2 \mathrm{CO}_{2}$ Bubbling}

We used the previously described tetrapolar cell in this assay. Fig. 8 shows the plot of the corresponding Rm\% as a function of time.

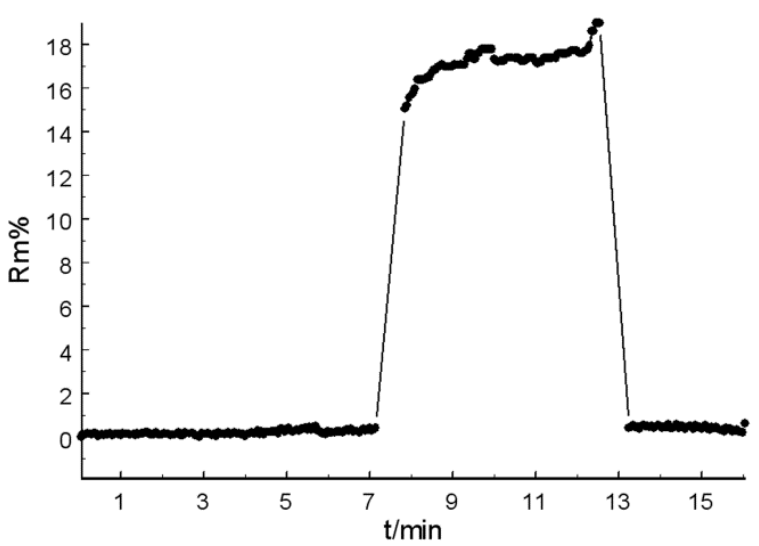

Fig. 8: Plot of the $\mathrm{Rm} \%$ as a function of time obtained from the bubbling of $\mathrm{CO} 2$ into the cell suspension. The black arrow indicates the start of bubbling. 
As shown in Fig. 8, the $\mathrm{CO}_{2}$ was insufflated at $7 \mathrm{~min}$. Unlike the gradual increase in the $\mathrm{Rm} \%$ shown in fig 3 and 4 , the $\mathrm{Rm} \%$ increased very rapidly from 0 to $19 \%$. This difference is attributed to the fact that few $\mathrm{CO}_{2}$ bubbles are released at the beginning of the fermentation and that this number increases as the fermentation progresses. In this $\mathrm{CO}_{2}$ bubbling experiment, the bubble flow rate is more intensive that the obtained in the fermentation processes, which explains the sudden increase in $\mathrm{Rm} \%$. This finding confirms the hypothesis that the $\mathrm{CO}_{2}$ bubbles produced by the fermentation are responsible for the observed changes in the $\mathrm{Rm} \%$.

\subsection{Monitoring of the sugar consumption using a blood glucose meter}

The bioimpedance measurement was performed simultaneously with the monitoring of the glucose concentration, which was achieved using a blood glucose meter. Fig. 9 shows plots of the $\mathrm{Rm} \%$ and the glucose concentration $[\mathrm{mM}]$ as a function of time.

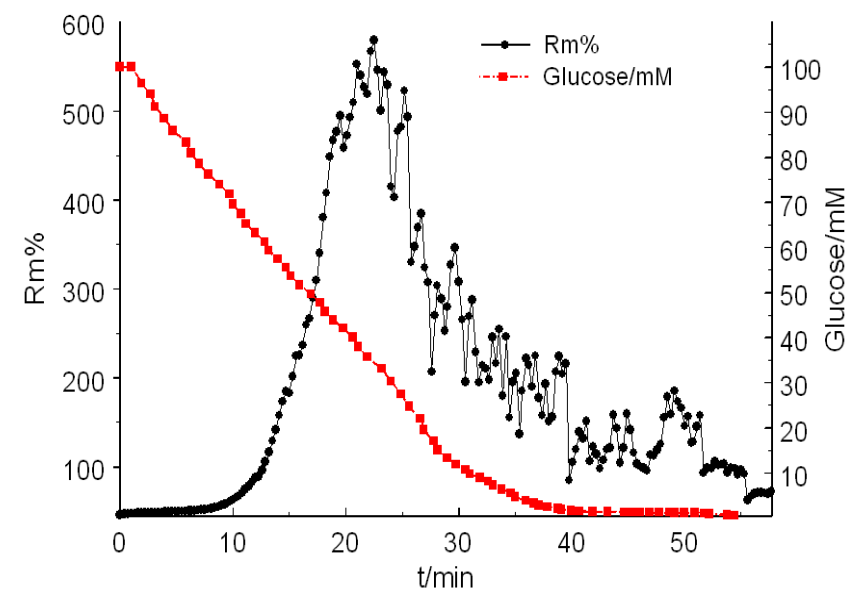

Fig. 9: Plot of the glucose concentration $(\mathrm{mM})$ as a function of time.

The experiment starts with the addition of glucose at $t_{0}=0$ min. As shown in the figure, the glucose concentration starts to decrease 1 minute after the addition of glucose, whereas $\mathrm{Rm} \%$ starts to change approximately 7 minutes after the addition of glucose (at this point, $20 \%$ of the glucose has been consumed).

\section{Discussion}

The method presented here is based on monitoring the consumption of sugar by measuring the effect of the $\mathrm{CO}_{2}$ bubbles produced by fermentation on the medium resistance. The advantages of this method over existing technologies are the following. i) As shown in Fig. 3, Rm starts to increase $7 \mathrm{~min}$ after the addition of glucose. This feature is very important because the assays used by other researchers [5] who have measured the alcoholic fermentation of yeast using impedance require approximately $200 \mathrm{~h}$, whereas the total assay times of our method are approximately 80 min and 100 min when using glucose and sucrose, respectively, as the carbon sources. In addition, Perez et al. detected the start of the fermentation after $50 \mathrm{~h}$ whereas we detected the start of the fermentation 7 minutes after the addition of the carbon source.

In Fig. 8 we can see that the changes in Rm depend on the amount of $\mathrm{CO}_{2}$ bubbles liberated by the fermentation. In the first few minutes of the fermentation, the amount of bubbles is low; however, as the fermentation progresses, this number increases until it induces changes in the $\mathrm{Rm}$. These results are in agreement with the resistance results obtained by Perez et al. These researchers reported that the resistance $\mathrm{Rm}$ started to increase approximately $50 \mathrm{~h}$ after the start of the experiment.

We worked at room temperature, which varied less than $1^{\circ} \mathrm{C}$ in all tests. In previous literature, it was stated that variations of $1{ }^{\circ} \mathrm{C}$ in temperature cause a variation of $2 \%$ in Rm (Felice, C. J., Ph.D Thesis, Universidad Nacional de Tucumán, Tucumán, Argentina, 1996). In our experiment we obtain variations of $700 \%$ and $1200 \%$ (glucose and sucrose respectively) so that a variation of $2 \%$ is negligible.

During sugar's metabolism the $\mathrm{pH}$ changes from 5.28 units, prior to sugar addition, to 4.29 when the fermentation ends. According to Firstenberg-Eden [8] a change of 2 units in $\mathrm{pH}$ represent a change of $14 \%$ in conductance approximated.

Again, this change is negligible compared to the changes observed in our experiments.

We did not measure the growth of yeast: our fermentation occurs without significant growth of yeast because $\mathrm{Rm} \%$ returned to its original value (its value prior to the addition of glucose or sucrose) after the sugar was consumed. If the yeast cells were experiencing growth, the Rm would stabilize at a different value [9]. To confirm that the yeast does not grow during the assay we perform a plate count and obtained $1,6 \times 10^{9} \mathrm{UFC} / \mathrm{mL}$ before the addition of sugar and $1,68 \times 10^{9} \mathrm{UFC} / \mathrm{mL}$ after the fermentation ends. ii) The proposed method is inexpensive because it only requires a gold electrode and does not require the use of enzymes [1]. In addition, the proposed method is quite simple because we applied an electric field with a known frequency and voltage instead of using other techniques, such as optical fiber refractometry and ultrasound.

It is important to note the difference in the plots obtained with different carbon sources (glucose and sucrose). The plot obtained using sucrose as the carbon source (Fig 4) showed that the increase in Rm was greater than that obtained using glucose as the carbon source (Fig $3)$. In addition, the time interval from the addition of the carbon source to the time at which the Rm returned to its original value (before the addition of the carbon source) was longer when sucrose was used as the carbon source than when glucose was used. Because sucrose is a disaccharide (glucose + fructose), the yeast cells use a cell wall invertase to catalyze the hydrolysis of sucrose into an equimolar mixture of glucose and fructose [10]. Saccharomyces cerevisiae is known to display a preference 
for glucose. Although fructose is used concomitantly with glucose, the latter is depleted first from the medium, which gives rise to a discrepancy between the amount of glucose and fructose consumed during a fermentation process [11]. Consequently, we can affirm that the plot obtained using sucrose as the carbon source follows the profiles of both fructose and glucose consumption. The comparison of the derivative plots showed that sucrose was consumed more rapidly than glucose.

Here we are measuring the effect of the $\mathrm{CO}_{2}$ bubbles, produced by the fermentation of sugar by yeast, over the medium resistance. We are not measuring the $\mathrm{CO}_{2}$ concentration in this work because we do not use and electrochemical sensor to perform this. The saline medium used here allows us to monitor only the consumption of sugar by yeast.

The use of a transport media, in order to avoid the multiplication of cells, could serve to perform a comparative analysis of the metabolism of sugars between different yeast's genera or subspecies

\section{Conclusions}

This paper presents a new method to monitor the sugar consumption in yeast. This method allows the real time monitoring of sugar's fermentation in yeast, by measuring the effect of $\mathrm{CO}_{2}$ bubbles over the medium resistance. The $\mathrm{CO}_{2}$ bubbles, obtained as a product of sugar fermentation, produce an increase in the medium resistance $(\mathrm{Rm})$ at 700 Hz. It proves to be possible to monitor the fermentation of different sugars (e.g., glucose or sucrose) and compare the speed at which they are consumed. The developed method has several advantages over existing technologies, such as its low cost, and reduced measurement time. A very important point is the fact that the observed changes are not due to the growth of yeast but only to the fermentation of sugars.

\section{Acknowledgments}

This work was supported by grants from the Agencia Nacional de Promoción Científica y Tecnológica (ANPCyT), the Consejo Nacional de Investigaciones Científicas y Técnicas (CONICET), and institutional funds from the Instituto Superior de Investigaciones Biológicas (INSIBIO) and Consejo de Investigaciones de la Universidad Nacional de Tucumán (CIUNT). The authors would like to thank Mr. Felix Colomo for completing the difficult task of designing the electrochemical cells used in this work.

\section{References}

1. Piermarini S, Volpe G, Esti M, Simonetti M, Palleschi G.Real time monitoring of alcoholic fermentation with low-cost amperometric biosensors. Food Chem.2011;127: 749-754. http://dx.doi.org/10.1016/j.foodchem.2011.01.008

2. Badotti F, Dário M, AlvesJr S, Cordioli MLA, Miletti LC, de Araujo P.S, Stambuk, BU. Switching the mode of sucrose utilization by Saccharomyces cerevisiae. Microb Cell Fact 2008;7:4. http://dx.doi.org/10.1186/1475-2859-7-4

3. Zosel J, Oelßner W, Decker M, Gerlach G, Guth U. The measurement of dissolved and gaseous carbon dioxide concentration.Meas. Sci. Technol. 2011; 22: 1-45. http://dx.doi.org/10.1088/0957-0233/22/7/072001

4. JiménezF, Vázquez J, Sánchez-Rojas JL, Barrajón N, Úbeda, J. Multi-purpose optoelectronic instrument for monitoring the alcoholic fermentation of wine. Sensors 2011 IEEE Conference, Ireland: $390-393$.

5. Pérez MA, Mu-iz R, de la Torre C, García B, Carleos CE, Crespo R, Cárcel LM. Impedance spectrometry for monitoring alcoholic fermentation kinetics under wine-making industrial conditions. XIX IMEKO World Congress Fundamental an Applied Metrology. September 6-11 2009 Lisbon, Portugal. 2574-2578.

6. Vogt H. The incremental ohmic resistance caused by bubbles adhering to an electrode. J Appl. Electrochem. 1983;13: 8788. http://dx.doi.org/10.1007/BF00615891

7. Bergman LW.Growth and Maintenance of Yeast. In: MacDonald, PN, editor. Two-Hybrid Systems: Method and Protocols. New Yersey: Humana Press; 2001. p. 9-14. http://dx.doi.org/10.1385/1-59259-210-4:009

8. Firstenberg-Eden R, Eden G. The Effect of $\mathrm{pH}$ changes of conductance and capacitance. In: Sharpe, AN, editor. Impedance Microbiology. New Yersey: Research Studies Press LTD, 1984. p. 62-63.

9. Felice CJ, Valentinuzzi ME. Medium and Interface Components in Impedance Microbiology. IEEE. Trans. Biomed. Eng.1999;46: 1483-1487. http://dx.doi.org/10.1109/10.804577

10. Andjelković U, Pićurić S, Vujčić Z. Purification and characterisation of Saccharomyces cerevisiae external invertase isoforms. Food. Chem. 2010; 120: 799-804. http://dx.doi.org/10.1016/j.foodchem.2009.11.013

11. Berthels NJ, Cordero Otero, RR, Bauer FF, Thevelein JM, Pretorius, IS. Discrepancy in glucose and fructose utilisation during fermentation by Saccharomyces cerevisiae wine yeast strains. FEMS Yeast Res. 2004:4: 683-689. http://dx.doi.org/10.1016/j.femsyr.2004.02.005 